\title{
Magnetic Force Nanoprobe for Direct Observation of Audio Frequency Tonotopy of Hair Cells
}

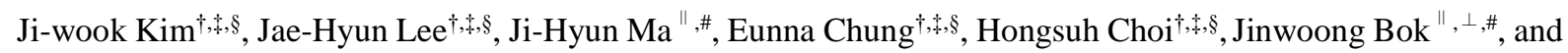
Jinwoo Cheon ${ }^{*}, \dagger, \star, \S$

${ }^{\dagger}$ Center for Nanomedicine, Institute for Basic Science (IBS), Seoul 03722, Republic of Korea.

*Yonsei-IBS Institute, Yonsei University, Seoul 03722, Republic of Korea.

${ }^{\S}$ Department of Chemistry, Yonsei University, Seoul 03722, Republic of Korea.

${ }^{\|}$Department of Anatomy, ${ }^{\perp}$ Department of Otorhinolaryngology, and ${ }^{\#}$ BK21 PLUS project for Medical Science, Yonsei University College of Medicine, Seoul 03722, Republic of Korea.

*Correspondence to: E-mail: jcheon@yonsei.ac.kr, Tel: +82-2-2123-5631, Fax: +82-2-2123-8229

\section{This file contains:}

Materials and Methods

Figures S1-S10

Supporting References 


\section{Preparations of magnetic nanoparticles}

Synthesis of magnetic nanoparticles. All chemical reactants are purchased from Sigma-Aldrich unless otherwise mentioned. Oleic acid and oleylamine are distilled under an argon atmosphere before use. The 40-nm cube-shaped magnetic nanoparticles are synthesized according to the protocol previously described in Noh et al. ${ }^{1}$ Briefly, iron(III) acetylacetonate $(2.0 \mathrm{mmol})$ and zinc(II) acetylacetonate (2.18 $\mathrm{mmol})$ are transferred into a $50 \mathrm{~mL}$ three-neck round-bottom flask containing oleic acid $(6.60 \mathrm{mmol})$ and benzyl ether $(30 \mathrm{~mL})$. The mixture is heated for 30 minutes at $290^{\circ} \mathrm{C}$ under an argon atmosphere and cooled to room temperature. Then, ethanol is added to the flask, and a black precipitate is observed. Then, the mixture is centrifuged, and the pellet is isolated and dispersed in toluene. The size of the synthesized cube-shaped magnetic nanoparticles are approximately $40 \mathrm{~nm}$ along one edge.

\section{Surface modification and labeling of the hair cell bundles with cube-shaped magnetic} nanoparticles. For the surface modification of MNPs, $20 \mathrm{mg}$ of MNPs and $1.4 \mathrm{~g}$ of tetramethylammonium are dissolved in $4 \mathrm{~mL}$ of butanol and sonicated. After $1 \mathrm{~h}$, the MNPs are collected by centrifugation (1,000 rcf, $1 \mathrm{~min}$ ) and redispersed in $20 \mathrm{~mL}$ of deionized water. $1.4 \mathrm{~g}$ sample of carboxymethyl $(\mathrm{CM})$ dextran (PK chemicals $\mathrm{A} / \mathrm{S}$ ) is added to the MNPs solution and heated at $70^{\circ} \mathrm{C}$ for $8 \mathrm{~h}$ (Figure S1). CM dextran-coated MNPs are collected by centrifugation (2,000 rcf, $5 \mathrm{~min}$ ) and redispersed in $10 \mathrm{mM}$ phosphate buffer solution. Wheat germ agglutinin (WGA) is conjugated on the surface of the CM dextran-coated MNPs for specific labeling of hair bundles. After dissolving of MNPs in $10 \mathrm{mM}$ phosphate buffer solution (pH 7.4), 1-ethyl-3-(3-(dimethylamino)propyl)carbodiimide (2 mM, Sigma Aldrich), N-hydroxysulfosuccinimide ( $5 \mathrm{mM}$, Thermo Scientific), and WGA (1 mg, Vector Laboratories) are added to the MNP solution and reacted for $40 \mathrm{~min}$ at room temperature. The final product is collected by centrifugation (final concentration of MNP: $0.5 \mathrm{mg} / \mathrm{ml}$ ). To confirm the labeling of MNPs on hair bundles, we analyze the co-localization of fluorescent dye-conjugated MNPs and Factin signals of the hair bundles using confocal microscopy. The hair bundles are stained using Alexa Fluor® 568 phalloidin (ThermoFisher Scientific) following the manufacturer's directions. Hair bundles are labeled with $0.1 \mathrm{mg} / \mathrm{mL}$ Alex 488 fluorescent dye (ThermoFisher Scientific) conjugated WGAMNPs by incubation in phosphate buffered saline (PBS) solution for $20 \mathrm{~min}$ and gentle washing three times. Fluorescence images showing hair cells in the whole tonotopy region from the apical to basal region of the basilar papilla are acquired using a confocal microscope (Zeiss LSM 700). 

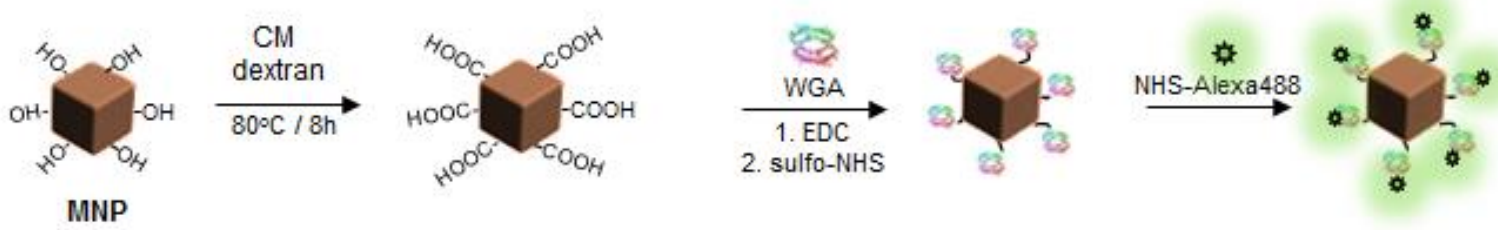

Figure S1. Scheme describing the preparation process of WGA-MNPs. 


\section{Preparations of chick hair cells}

Sample preparation from a chick inner ear. Post-natal chicks (day 0-2, Hong-ik Hatchery in Pyeongtaek, Gyeonggi-do, South Korea) are used for the experiments in the current study due to their well-developed tonotopic organization at the neonatal stage ${ }^{2,3}$. Animals are managed following the Guidelines for the Care and Use of Laboratory Animals of Yonsei University College of Medicine. To maintain tissue viability and functionality in ex vivo experiments, the whole inner ear is immediately immerged and incubated in extracellular buffer (ECB) during dissection. The ECB is composed of 87 $\mathrm{mM} \mathrm{NaCl}, 0.5 \mathrm{mM} \mathrm{KCl}, 0.5 \mathrm{mM} \mathrm{CaCl}_{2}, 1.25 \mathrm{mM} \mathrm{NaH}_{2} \mathrm{PO}_{4}, 2.0 \mathrm{mM}$ ascorbate, $2.0 \mathrm{mM}$ creatine, 6.0 mM Na-pyruvate, $75 \mathrm{mM}$ sucrose, $25 \mathrm{mM}$ D-glucose, and $10 \mathrm{mM}$ HEPES (pH 7.4, 310-320 mOsm) ${ }^{4}$. The brain, soft tissue, cartilage and bone are serially removed from the whole inner ear tissue, and then the basilar papilla (BP) is isolated. The BP is incubated in the protease solution ( $50 \mu \mathrm{g} / \mathrm{ml}$, Type XXIV, Sigma Aldrich) $)^{5}$ for 15 minutes at room temperature. The BP is washed three times for 3 min and incubated in $2 \%$ bovine serum albumin solution to block nonspecific targeting. The tissue is attached on the plastic cover slip using dental glue (Histocary ${ }^{\circledR}$ L, B BRAUN) and briefly washed with the ECB.

Morphological analysis of hair bundles. The morphology of hair bundles at different regions along the tonotopy is investigated by scanning electron microscopy (SEM). For fixation, the basilar papilla is dissected in $2 \%$ paraformaldehyde $/ 2.5 \%$ glutaraldehyde in $0.1 \mathrm{M}$ sodium cacodylate buffer $(\mathrm{pH} 7.4)$ for $2 \mathrm{~h}$ at room temperature. The tissue is further incubated overnight in $2.5 \%$ glutaraldehyde/ $0.1 \mathrm{M}$ sodium cacodylate buffer with $3.5 \%$ sucrose solution and $2 \mathrm{mM} \mathrm{CaCl}_{2}$. Post-fixation is performed using the $\mathrm{OsO}_{4} /$ thiocarbohydrazide (OTOTO) protocol ${ }^{3,6}$. After post-fixation, the samples are dehydrated through gradient concentrations of ethanol solutions $(20 \%, 40 \%, 60 \%, 70 \%, 80 \%, 90 \%, 95 \%$, and $100 \%)$, and the dehydrated specimens in isoamyl acetate are dried following the method of critical point drying (HCP-2, Hitachi). The samples are mounted on a stub holder and coated with platinum in $5 \mathrm{~nm}$ thickness for SEM imaging.

Viability analysis of hair bundles. To demonstrate whether hair cells in the BP are viable and maintains their transductive functionality of ion channels during the experiments, the viability of the samples are analyzed using FM1-43 ( $N$-(3-triethylammoniumpropyl)-4-(4-(dibutylamino) styryl) pyridinium dibromide, ThermoFisher Scientific), as described in a previous study ${ }^{7,8}$. Briefly, the tissue prepared from the basilar papilla is incubated in $5 \mu \mathrm{M}$ FM1-43 for 10 seconds and washed three times in ECB. Then, the tissue is mounted in Prolong ${ }^{\circledR}$ gold antifade (ThermoFisher Scientific) and is covered by a coverslip (EMS, Thickness \#0). The basilar papilla is observed using a confocal microscope (Zeiss, LSM 780) with a 100X oil immersion lens (Figure S2). 


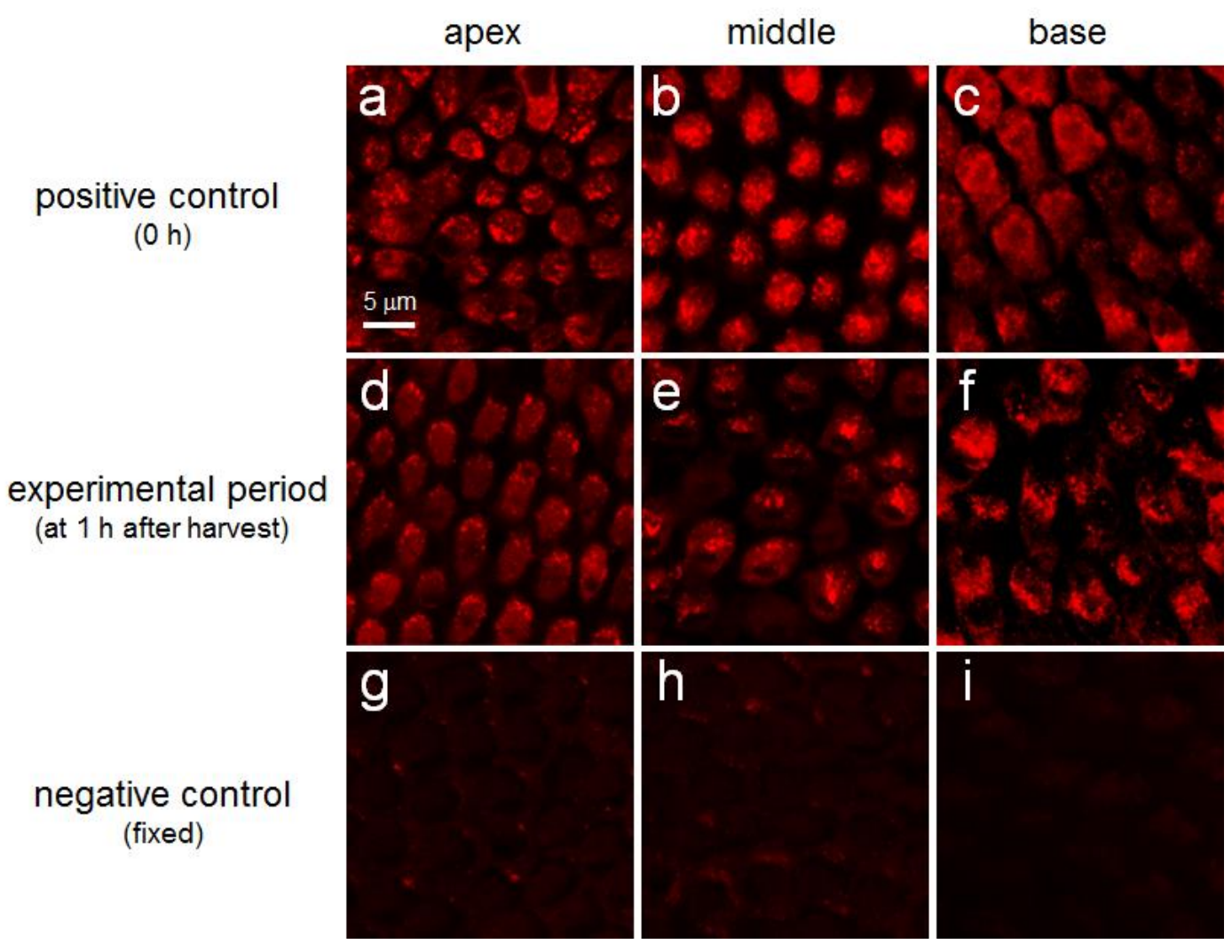

Figure S2. Functionality of ion channels in hair cells at different locations along the tonotopy. FM1-43 fluorescence measured at $0 \mathrm{~h}$ (freshly prepared hair cells) (a-c) and $1 \mathrm{~h}$ (termination time point of the experiments in this study) (d-f). ( $g-i)$ FM1-43 fluorescence measured at hair cells following fixation using paraformaldehyde (4\%). 


\section{Fabrication and characterization of the microelectromagnet}

Fabrication of the microelectromagnet. The microelectromagnet used in all experiments of the current study is constructed based on a previously reported procedure, with minor modifications ${ }^{9}$. The permalloy magnetic probe tip is composed of $75 \% \mathrm{Ni}$ and $25 \% \mathrm{Fe}(10 \mu \mathrm{m}$ probe tip diameter and 25 $\mathrm{mm}$ probe length, DSF System). The permalloy magnetic probe tip is rounded (1,200 turns) with copper wire $(50 \mu \mathrm{m}$ diameter, Elfa Distrelec). The resistance of the electromagnetic coil is $70 \Omega$. The electromagnetic coil is connected to a signal generator (AFG3022C, Tektronix). The microelectromagnet is housed in a water-circulation jacket to prevent overheating.

Calculation of the magnetic field strength. The magnetic field strength of the microelectromagnet is calculated by FEMM simulation (FEMM 4.2, David Meeker) ${ }^{10}$. The detailed parameters for simulation are 1) diameter of copper wire $=50 \mu \mathrm{m}, 1,200$ turns; 2) length, diameter, and tip-end size of the permalloy probe $=25 \mathrm{~mm}, 0.8 \mathrm{~mm}$, and $10 \mu \mathrm{m}$, respectively; and 3) current in the microelectromagnet $=50 \mathrm{~mA}$. The magnetic field strength is $180 \mathrm{mT}$ at $5 \mu \mathrm{m}$ from the magnet (Figure S3).

a

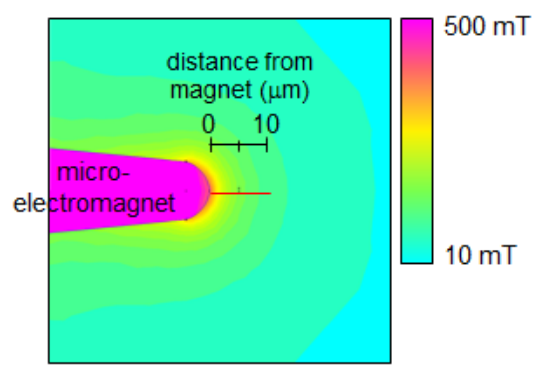

b

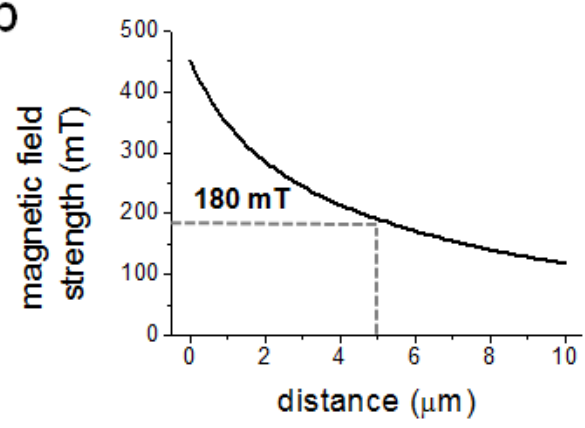

Figure S3. (a) Calculated magnetic field strength around the tip-end of the microelectromagnet. (b) distance-dependent magnetic field strength simulated by using FEMM. 


\section{Calculation of the force applied to the single hair bundles}

To calculate the force exerted on a cube-shaped MNP by the magnetic field gradient, three measured values are used: one-dimensional $\mathrm{dB} / \mathrm{dx}$, the magnetization of the particle $(\mathrm{emu} / \mathrm{g})$, and the size of the particle. The mass of a single cube-shaped magnetic nanoparticle is calculated to be $6.6 \times 10^{-16} \mathrm{~g}$ on the basis of the known volume of a cube with a $40 \mathrm{~nm}$ edge and the mean density of ferrite materials (5300 $\mathrm{kg} \cdot \mathrm{m}^{-3}$ ). Assuming that nanoparticles are magnetically saturated in the applied field gradient, the magnetic moment of a single cube-shaped magnetic nanoparticle is $1 \times 10^{-13} \mathrm{emu}\left(=1 \times 10^{-16} \mathrm{~A} \cdot \mathrm{m}^{2}\right)$ on the basis of the values of the saturation magnetization of $162 \mathrm{emu} \cdot \mathrm{g}^{-1}$ (Figure S4a). We have previously reported the magnetic field gradient map of our magnetic probe tip which is measured by using Stokes drag force ${ }^{11}$. The velocity of $1 \mu \mathrm{m}$ magnetic beads which attracted to probe tip end is obtained at $80 \%$ glycerol. From the Stokes law, $\mu \vec{\nabla} B=3 \pi \eta d \vec{v}$, where $\mu=6.5 \times 10^{14} \mathrm{~A} \cdot \mathrm{m}^{2}$ is the magnetic moment of the magnetic bead, $\eta=6.14 \times 10^{-2} \mathrm{~Pa} \cdot \mathrm{s}$ is the $80 \%$ glycerol solution viscosity, $\mathrm{d}=1.1 \mu \mathrm{m}$ is mean bead diameter, and $\vec{v}$ is the velocity of micro beads, the magnetic field gradient $(\vec{\nabla} B)$ can be calculated. The magnetic field gradient is $5,000 \mathrm{~T} \cdot \mathrm{m}^{-1}$ at $5 \mu \mathrm{m}$ distance from the magnet end. The force exerted is a product of the field gradient and the magnetic moment ${ }^{12}$; therefore, a cube-shaped MNP experiences ca. $0.2 \mathrm{pN}$ of force at $5,000 \mathrm{~T} \cdot \mathrm{m}^{-1}$ (distance from magnet: $5 \mu \mathrm{m}$, Figure $\mathrm{S} 4 \mathrm{~b}$ ). The overall surface area of the single hair bundle at the apical, middle, and basal regions are $47.1,49.2$, and $46.8 \mu \mathrm{m}^{2}$, respectively, from SEM images taken in the current study and the morphology simulation based on previously reported results (Figure $S 4 c-d)^{13}$. The number of MNPs on $1 \mu \mathrm{m}^{2}$ hair bundle surface is $29 \pm 3$ particles according to the SEM study $(n=6)$ (Figure S4e). The average number of MNPs on a single hair bundle in each region is thus approximately 1,400, and the applied force is approximately $280 \mathrm{pN}$ at $5 \mu \mathrm{m}$ from the magnet. 
a

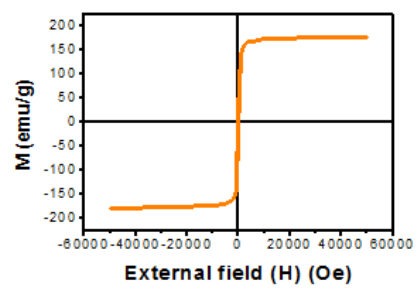

b

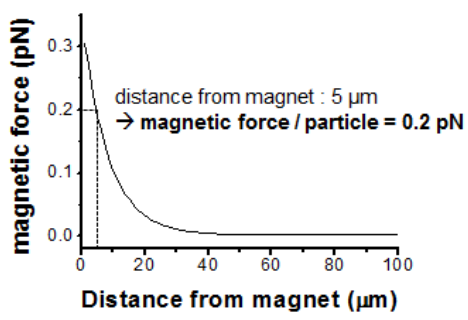

C

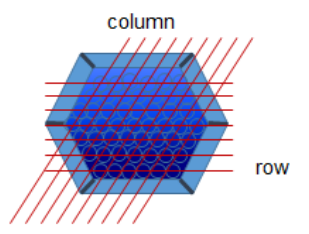

d

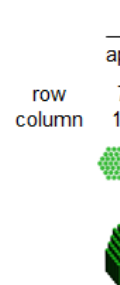

number of stereocilia

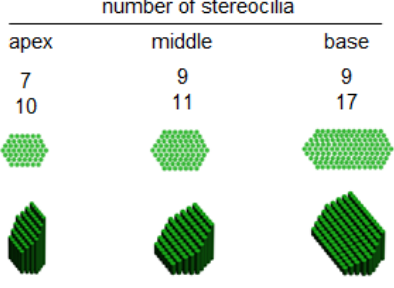

e

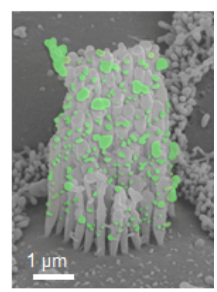

Figure S4. Mechanical force applied to a single hair bundle. (a) Hysteresis curve of cube shaped magnetic nanoparticles which is acquired using a superconducting quantum interference device (SQUID) magnetometer. (b) The force calibration of a single magnetic nanoparticle. (c) The array of the stereocilia in a hair bundle. (d) Simulated morphologies of hair bundles and the numbers of the stereocilia in the single row and the column in the apical, middle, and basal regions. (e) A representative SEM image of the MNPs (green, pseudo color mapping)-bound hair bundle in the middle region.

\begin{tabular}{|c|c|c|c|c|c|}
\hline & \multirow{2}{*}{$\begin{array}{l}\text { No. of stereocilia } \\
\text { in single hair } \\
\text { bundle }\end{array}$} & \multicolumn{2}{|c|}{ Length of stereocilia $(\mu \mathrm{m})$} & \multirow{2}{*}{$\begin{array}{l}\text { Diameter of } \\
\text { stereocilia } \\
(\mu \mathrm{m})\end{array}$} & \multirow{2}{*}{$\begin{array}{l}\text { Surface area } \\
\text { per single hair } \\
\text { bundle }\left(\mu \mathrm{m}^{2}\right)\end{array}$} \\
\hline & & $\begin{array}{c}\text { Shortest } \\
\text { stereocilia }\end{array}$ & $\begin{array}{c}\text { Longest } \\
\text { stereocilia }\end{array}$ & & \\
\hline $\begin{array}{c}\text { Apical hair bundle } \\
(20 \%)\end{array}$ & 51 & 1.7 & 3.7 & 0.26 & 47.1 \\
\hline $\begin{array}{c}\text { Middle hair bundle } \\
(50 \%)\end{array}$ & 79 & 0.8 & 2.8 & 0.33 & 49.2 \\
\hline Basal hair bundle (70\%) & 133 & 0.5 & 2.3 & 0.32 & 46.8 \\
\hline
\end{tabular}

Table S1. Surface area of the hair bundles calculated based on the morphology analysis of chick hair bundles in Figure S4d. The obtained surface of the hair bundles were 47.1, 49.2, and $46.8 \mu \mathrm{m}^{2}$ at the apical, middle, and basal region hair bundles, respectively. Total numbers of MNPs on the surface of the hair bundle: ca. 1,400 particles. The applied mechanical force to the single hair bundle: ca. $280 \mathrm{pN}$. 


\section{Magnetic stimulation of a hair bundle and high speed imaging}

Magnetic stimulation of a hair bundle. A magnetic probe tip is positioned close to the MNP-labelled hair bundle using a motorized micromanipulator (Sutter instrument). The distance from the probe tip to the bundle is adjusted to be within $5 \mu \mathrm{m}$. A current of $50 \mathrm{~mA}$ is applied to magnetize the permalloy probe, and $-10 \mathrm{~mA}$ is applied to demagnetize the permalloy probe.

Recording hair bundle displacement. The motion of the hair bundles is imaged with an optical microscope (Nikon, Eclipse Ti-U) equipped with a water immersion objective (60X, $0.95 \mathrm{NA}$ ) and a complementary metal oxide semiconductor (CMOS) camera (Photron, Fastcam Mini UX100) at an imaging rate of 20,000 frames per second. In order to trace the oscillatory movement of hair bundle, a Gaussian-fit algorithm was utilized to mathematically estimate the axial position of single hair bundles to overcome the diffraction limit ${ }^{14,15}$. In brief, to trace the motion of the hair bundles, the horizontal line scan on hair bundle was derived through the images in each frame, and a Gaussian distribution was fitted on the intensity profile of the bundle to extract the center position (Figure S5a,b). To improve the signal-to-noise ratio of the bundle position, 1) remove the low level signal from the images, (Figure S5a), 2) vertically adjacent pixel rows were also tracked and averaged (Figure S5b), which provides the exact position of the bundle in nanometer scale of spatial resolution (Figure S5c).
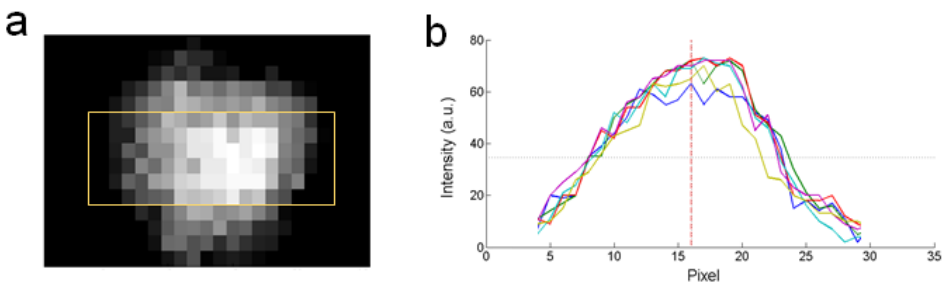

C
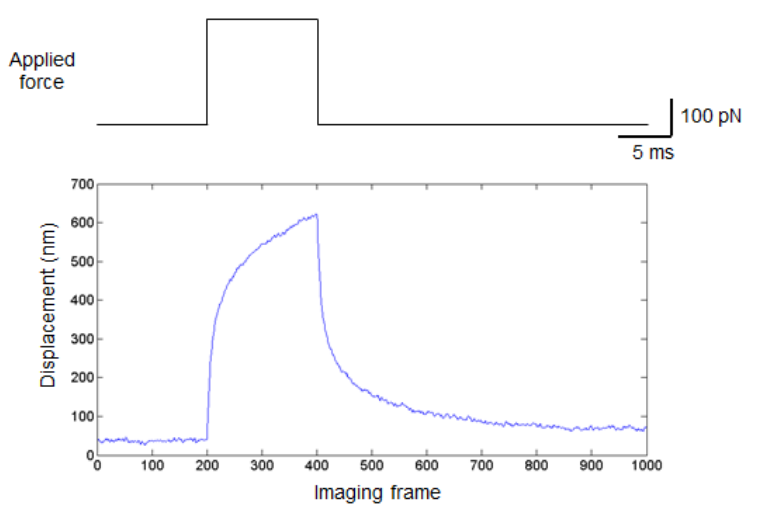

Figure S5. Tracking the hair bundle displacement. (a) Top down view of the hair bundle. Low level signals are removed by enhancing the contrast of the images. (b) Gaussian-fits of the intensity profile of the bundle from the yellow box in a. (Red dotted line: center position of a hair bundle) (c) Applied force (top) and analyzed hair bundle displacements. 


\section{Calculation of the stiffness of the hair bundles}

The stiffness of the hair bundles is calculated from the applied force-bundle displacement relationship plots. Step increases of the magnetic pulses (22.5 to $180 \mathrm{mT}$ ) are applied to MNP-labeled hair bundles. The calculated forces are 35 to $280 \mathrm{pN}$. Figure S6a shows the applied force (i) and the displacement of hair bundles at the apical, middle, and basal regions (ii). From the bundle displacements, the applied force-bundle displacements relationship is plotted, and the stiffness of the hair bundles, which is the slope of the plot, is obtained (Figure S6b). Next, the stiffness of the hair bundles is measured along the tonotopic axis (Figure S6c).

a i applied

ii bundle displacement

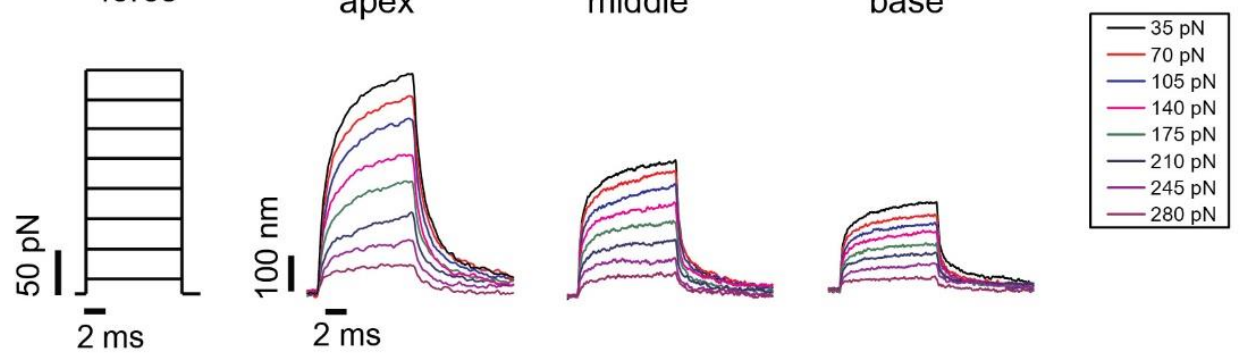

b

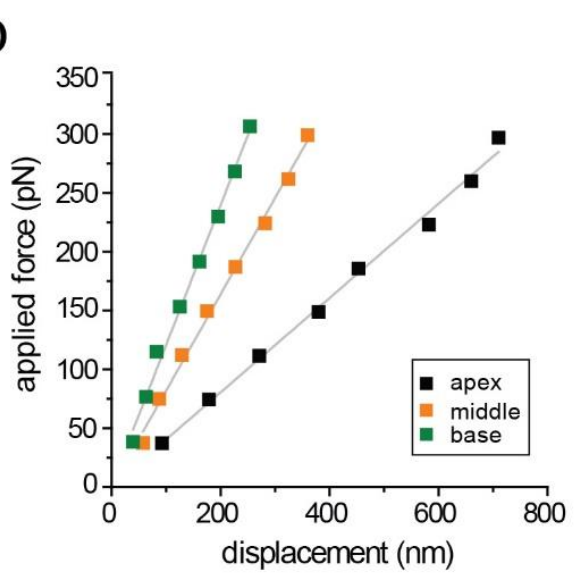

C

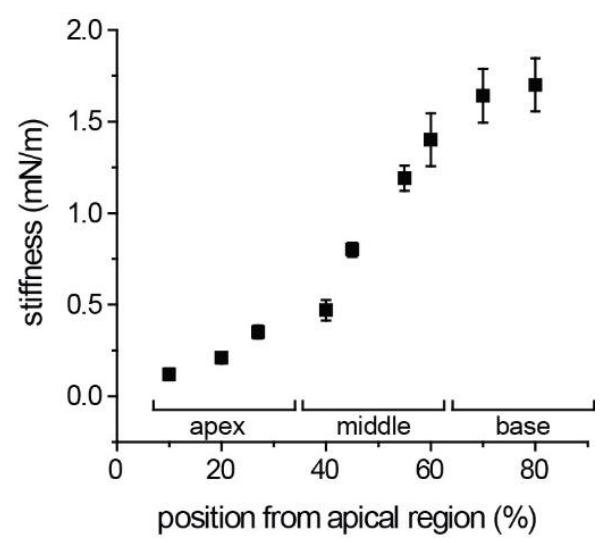

Figure S6. Stiffness of hair bundles measured by MFN. (a) Applied force to hair bundles (i) and corresponding displacement of hair bundle at apical, middle, and basal regions of cochlea (ii). (b) The stiffness of hair bundles which is a slope of the force-displacement relationship plots. (c) Stiffness of hair bundles measured along the tonotopic axis of cochlea. 


\section{Measurement of time constants in hair bundle recovery trajectories}

Time constant measurement of the glass fiber. The time constants are obtained by fitting the recovery trajectory of hair bundles with an exponential function using the Origin 8.0 software (Origin Lab). For model comparison between the first-, second-, and third-order exponential functions, F-tests are performed using the Origin software, and the second-order exponential (Equation 1) model is the bestfitted function in all tests, with $\mathrm{p}$ values below 0.001 .

$$
\mathrm{D}(\mathrm{t})=\mathrm{A}_{1} \exp \left(\frac{-\mathrm{t}}{\tau_{\text {fast }}}\right)+\mathrm{A}_{2} \exp \left(\frac{-\mathrm{t}}{\tau_{\text {slow }}}\right)+\mathrm{B}
$$

In addition, the recovery trajectories are fitted by second-order exponential fitting only when the $\mathrm{R}^{2}>$ 0.99 and $\tau_{\text {slow }}>1.1 \mathrm{~ms}$ (Figure S7a). In contrast, hair cells following incubation for $2 \mathrm{~h}$ at room temperature, which has lost the bundle activity and considered to be dead, exhibit single time constants $(\tau=0.51 \mathrm{~ms})$ (Figure $\mathrm{S} 7 \mathrm{~b})$. This value is in ranges of $\tau_{\text {fast }}(0.3 \sim 0.9 \mathrm{~ms})$. The recovery trajectory of the glass fiber with similar stiffness $(0.47 \mathrm{mN} / \mathrm{m})$ to the hair bundle is fitted only with the first-order exponential function $\left(\mathrm{R}^{2}=0.99, \tau=0.59 \mathrm{~ms}\right.$ ) (Figure $\mathrm{S} 7 \mathrm{c}$ ).

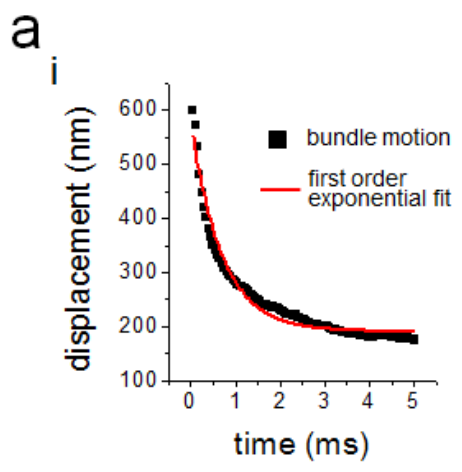

b

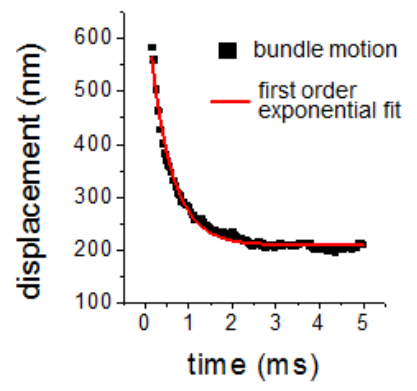

ii

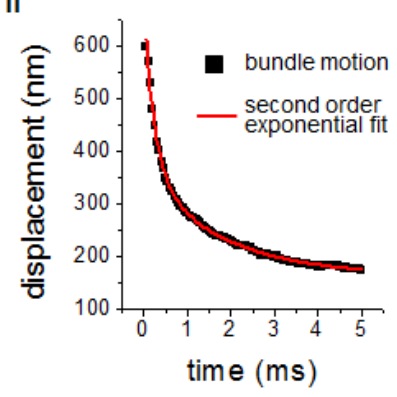

C

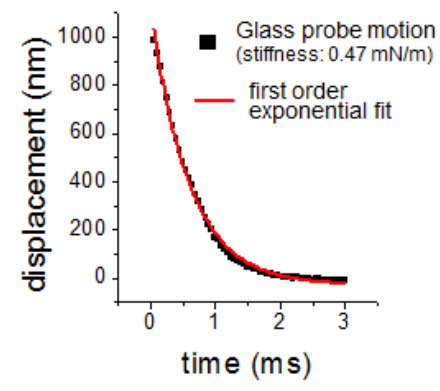

Figure S7. Time constant measurement in recovery motion of the hair bundle and flexible glass fiber. (a) Measurement of the time constant in the recovery motion of the hair bundles. The hair bundle motions in middle region and fitted curve at first (i) and second (ii) order exponential function. (b) Recovery trajectory of hair cells incubated for $2 \mathrm{~h}$ at room temperature and fitted curve to first order exponential function. (c) Recovery trajectory of a glass fiber and fitted curve to first order exponential function. 
Time constant measurement of hair bundles with different buffer conditions. 1) Calcium concentration-dependent time constant: Calcium concentrations of $0.5,1.0$, and $1.5 \mathrm{mM}$ ECB are applied for 10 minutes before observation of the displacement of the hair bundle. The time constants are measured with the same method described in the manuscript. 2) The effect of butanedione monoxime (BDM) on the time constant: Before BDM is applied to the basilar papilla, the displacement of the hair bundle in normal ECB is measured. Then, the buffer is exchanged to $10 \mu \mathrm{M}$ BDM for 10 minutes, and the time constants are derived from displacement of the hair bundle. 3) The effect of gentamicin on the time constant. To test the effect of ion channel blocking on the recovery dynamics of hair bundle, $\mathrm{Ca}^{2+}$ channels were blocked by a well-known channel blocker, gentamicin. We have applied $80 \mu \mathrm{M}$ of gentamicin and recorded the bundle displacement upon the same bundle stimulation at $10 \mathrm{~ms}$ magnetic pulse. In this condition, both bundle displacement and $\tau_{\text {slow }}$ decreased to $50 \mathrm{~nm}$ and $0.9 \mathrm{~ms}$, respectively (Figure S8). However, value of $\tau_{\text {fast }}$ didn't show any significant changes.

\section{a}

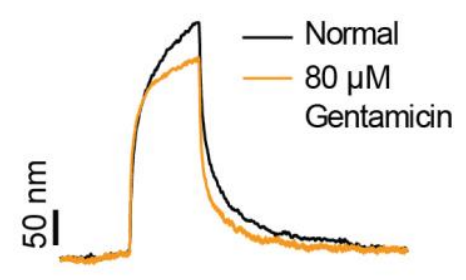

b

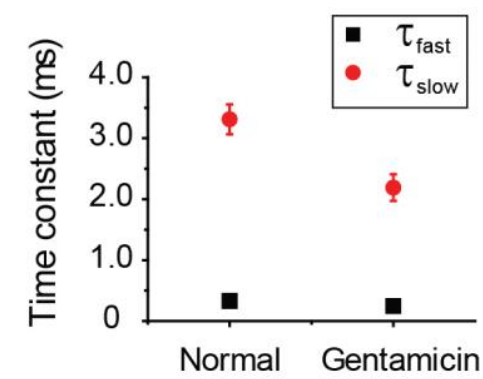

Figure S8. Gentamicin effects on the time constants ( $\tau_{\text {slow }}$ and $\left.\tau_{\text {fast }}\right)$ in the recovery dynamics of hair bundles. Hair bundle displacement (a) and measured time constants (b) on $80 \mu \mathrm{m}$ gentamicin treatment. $(n=8)$. 


\section{Calculation of recovery distance of hair bundles}

The recovery distance is the displacement of the hair bundle recovered from the deflected state in a given time, which is semi-empirically calculated (Equation 2) by using the second-order exponential function in which $\tau_{\text {slow }}, \tau_{\text {fast }}$ and other variables are substituted with empirically measured values.

$$
\text { Recvery distance }=A_{1}\left(\exp \left(\frac{-t}{\tau_{\text {fast }}}\right)-\exp \left(\frac{-t^{\prime}}{\tau_{\text {fast }}}\right)\right)+A_{2}\left(\exp \left(\frac{-t}{\tau_{\text {slow }}}\right)-\exp \left(\frac{-t^{\prime}}{\tau_{\text {slow }}}\right)\right)
$$

where $t$ and $t$ ' are time, and $\tau_{\text {fast }}, \tau_{\text {slow }}, A_{1}$, and $A_{2}$ are constants. All parameters, including $\tau_{\text {fast }}, \tau_{\text {slow }}, A_{1}$, and $A_{2}$, are experimentally derived from the exponential curve fitting of the bundle recovery motions of the apical $(n=7)$, middle $(n=7)$, and basal hair bundles $(n=7)$ with a single magnetic pulse with different durations (from 0.1 to $10 \mathrm{~ms}$ ). The measured $\tau_{\text {fast }}, \tau_{\text {slow }}, \mathrm{A}_{1}$, and $\mathrm{A}_{2}$ are plotted against the magnetic pulse duration. (Figure S9). Periodic magnetic pulses of 10, 5, 1, 0.5, 0.33, 0.25, 0.17, and 0.1 $\mathrm{ms}$ are corresponded to 50,100, 500, 1000, 1500, 2000, 3000, and $5000 \mathrm{~Hz}$, respectively. Therefore, the recovery distance of the hair bundle at a certain frequency is calculated using the parameters measured at the corresponding magnetic pulse durations.

Figure S10 shows the calculated recovery distances at different stimulation frequencies, which show several key points to address: 1) overall, higher bundle recovery distances are estimated at lower stimulation frequencies because there is enough time for the bundle to recovered at lower frequency than at higher frequency; and 2) more importantly, the curves of the trend explained in 1) is slightly different at the apical, middle, and basal hair bundles, which shows notable changes in bundle recovery distance. For example, the recovery distance of the apical hair bundle is $c a .450 \mathrm{~nm}$, which is relatively larger than that of the middle and basal regions in the range of 50 to $500 \mathrm{~Hz}$ (gray region), whereas the middle and basal hair bundles have the longest recovery distances at 500 to 2,000 $\mathrm{Hz}$ (orange region) and 2,000 to $5,000 \mathrm{~Hz}$ (green region), respectively. 
a

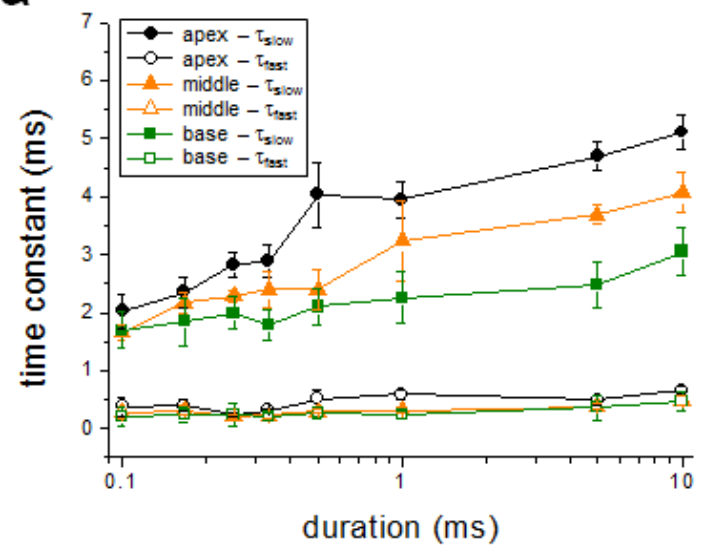

b

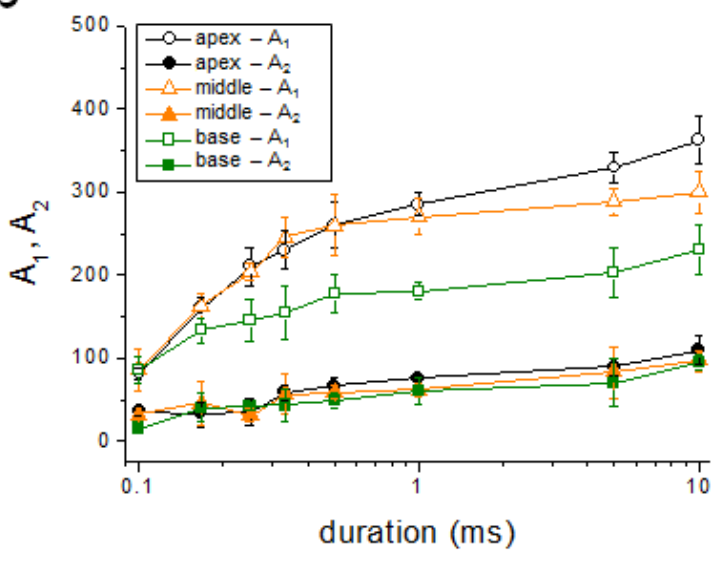

Figure S9. Derived parameters of $\tau_{\mathrm{fast}}, \tau_{\text {slow }}(\mathrm{a})$ and $\mathrm{A}_{1}, \mathrm{~A}_{2}(\mathrm{~b})$ at various durations of magnetic pulses for calculating recovery distance at certain frequency.

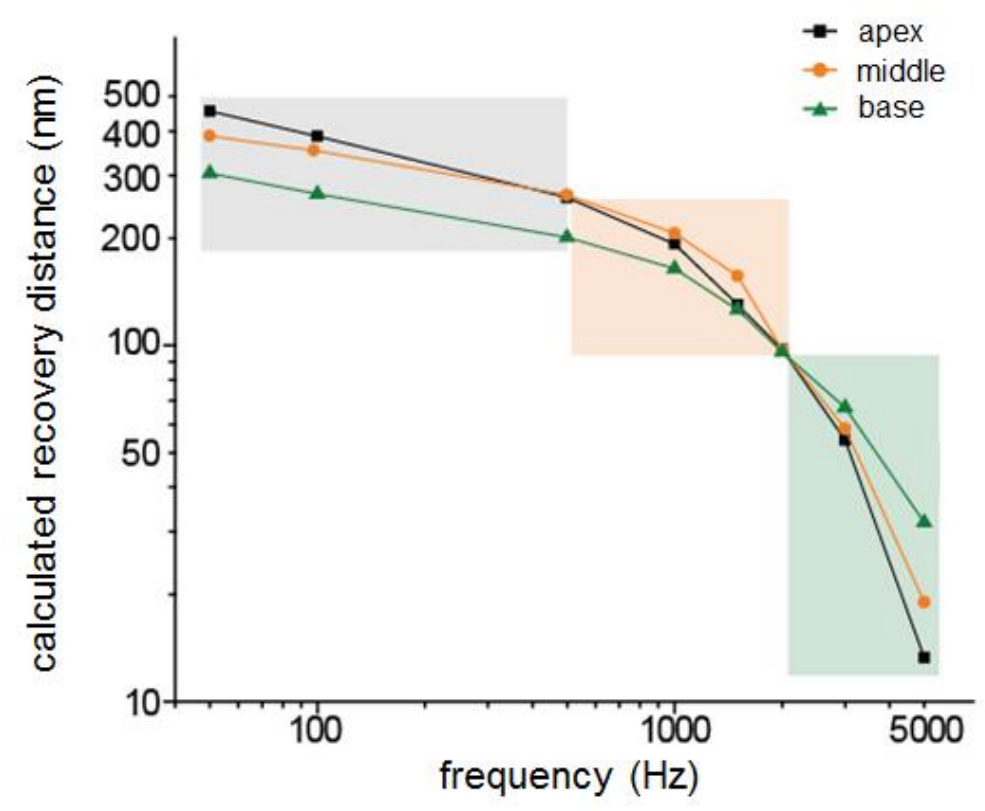

Figure S10. The calculated recovery distance of hair bundles at apical, middle, and basal regions with different stimulation frequencies. 


\section{References}

(1) Noh, S.-h.; Na, W.; Jang, J.-t.; Lee, J.-H.; Lee, E. J.; Moon, S. H.; Lim, Y.; Shin, J.-S.; Cheon, J. Nano Lett. 2012, 12, 3716-3721.

(2) Manley, G.A.; Brix, J.; Kaiser, A. Science 1987, 237, 655-656.

(3) Jones, T.A.; Jones, S.M.; Paggett, K.C. J. Neurophysiol. 2006, 96, 128-141.

(4) Spinelli, K.J.; Gillespie, P.G. PLoS One 2012, 7, e51874.

(5) Son, E.J.; Ma, J.H.; Ankamreddy, H.; Shin, J.O.; Choi, J.Y.; Wu, D.K.; Bok, J. Proc. Natl. Acad. Sci. USA. 2015, 112, 3746-3751.

(6) Hunter-Duvar, I.M. Acta Otolaryngol Suppl. 1978, 351, 3-23.

(7) Si, F.; Brodie, H.; Gillespie, P.G.; Vazquez, A.E.; Yamoah, E. N. J. Neurosci. 2003, 23, 1081510826.

(8) Lelli, A.; Asai, Y.; Forge, A.; Holt, J.R.; Geleoc, G.S. J. Neurophysiol. 2009, 101, 2961-2973.

(9) Matthews, B.D.; LaVan, D.A.; Overby, D.R.; Karavitis, J.; Ingber, D.E. Appl. Phys. Lett. 2004, 85, 2968-2970.

(10) Meeker, D.C. IEEE Trans. Magn. 2014, 50, 7400609.

(11) Lee, J.-H.; Kim, J.-w.; Levy, M.; Kao, A.; Noh, S.-h.; Bozovic, D.; Cheon, J. ACS Nano 2014, $8,6590-6598$.

(12) Boyer, T. H. Am. J. Phys 1988, 56, 688-692.

(13) Tilney, L.G.; Saunders, J.C. J. Cell Biol. 1983, 96, 807-821.

(14) Heintzmann R.; Ficz, G. Brief. Funct. Genomics Proteomics. 2006, 5, 289-301.

(15) Ramunno-Johnson, D.; Strimbu, C.E.; Fredrickson, L.; Arisaka, K.; Bozovic, D. Biophys J. 2009, 96, 1159-1168. 